\title{
壳聚糖及其衍生物负载过渡金属催化剂在有机反应中的应用进展
}

\author{
王硕周宏勇宋沙沙王家喜* \\ (河北工业大学化工学院 天津 300130)
}

\begin{abstract}
摘要 壳聚糖因其与金属化合物的良好络合性能成为金属催化剂的优良载体，壳聚糖及其衍生物负载金属及金属络合 物的催化性能研究近年来引起人们的广泛关注. 以反应类型为主线, 对这类催化剂在不对称催化、烯丙基取代、偶联 反应、加成反应、氧化反应、还原反应等各类有机反应中的应用进行了综述.
\end{abstract}

关键词 壳聚糖; 壳聚糖衍生物; 过渡金属; 催化反应; 不对称反应

\section{Applications of Chitosan and Its Derivatives Supported Transitional Metal Catalysts in Organic Reactions}

\author{
Wang, Shuo Zhou, Hongyong Song, Shasha Wang, Jiaxi* \\ (School of Chemical Engineering, Hebei University of Technology, Tianjin 300130)
}

\begin{abstract}
Due to its good coordination ability to the metal compounds, chitosan and its derivatives can be used as a kind of good catalysts carrier. Recently, the catalytic properties of chitosan and its derivatives supported metal catalyst have been attracted more attentions. Based on the kind of reactions, the applications of chitosan and its derivatives supported metal catalysts in the catalytic asymmetric reaction, allylic substitution, coupling, addition, oxidation, reduction and some other reactions are reviewed.
\end{abstract}

Keywords chitosans; chitosan derivatives; transition metal; catalytic reaction; asymmetric reaction

壳聚糖[(1,4)-2-氨基-2-脱氧- $D$-葡萄糖, CS] 是甲壳 素脱乙酰化的产物, 易溶于稀酸, 可以制备出各种形态 如粉末 ${ }^{[1]}$ 、凝胶 ${ }^{[2 \sim 8]}$ 、薄膜 ${ }^{[9 \sim 12]}$ 、纤维 ${ }^{[13 \sim 16]}$ 等, 可作为 $\mathrm{SiO}_{2} 、 \mathrm{Al}_{2} \mathrm{O}_{3} 、 \mathrm{Fe}_{3} \mathrm{O}_{4}$ [17 19]等氧化物的载体. 壳聚糖结构 中含有大量的 $\mathrm{NH}_{2}$ 和 $\mathrm{OH}$ 官能团, 可用于化学修饰反应 (如制备壳聚糖衍生物及络合金属离子). 近年来壳聚糖 作为生物高分子广泛应用于 $\mathrm{Pt} 、 \mathrm{Ru} 、 \mathrm{Rh}$ 或 $\mathrm{Pd}$ 等贵金属 催化剂的载体 ${ }^{[20]}$, 其负载金属催化剂已经广泛应用在 氧化、还原、加成等反应中, 表现出优良的催化性 能 ${ }^{[21 ~ 23]}$. Guibal ${ }^{[24]}$ 和 Macquarrie 等 ${ }^{[25]}$ 对早期壳聚糖及其 衍生物负载的金属催化剂的形态结构、催化反应的研究 已经进行了详细地综述. 目前壳聚糖及其衍生物在催化 反应中主要用作催化剂的载体, 但其分子中的氨基可作 为有机碱, 在新近的报道中其本身也可以作为催化剂催 化反应 ${ }^{[26,27]}$. 本文以反应类型为主线, 对近年来壳聚糖 及其衍生物负载金属催化剂催化有机反应进行了综述.

\section{1 不对称反应}

壳聚糖具有天然的手性特征，在不对称催化中能够 起到重要作用. 研究壳聚糖及其衍生物负载金属催化剂

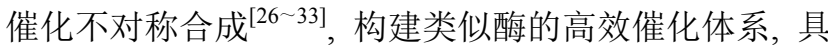
有重要意义.

Gagnon 等 ${ }^{[28]}$ 用改性壳聚糖与甲基异丙基苯二氯化 钉组成催化体系, 制备出以壳聚糖为配体的催化剂 $\mathbf{1}$, 用于苯乙酮类不对称催化还原反应。当以甲醇/异丙醇 $(V: V=9: 1)$ 为溶剂, 异丙醇钠为碱时, 苯乙醇的产率 高达 $81 \%$, 产物 $e e$ 值为 $68 \%$. 尽管该反应的对映体选择 性不是特别高, 但其作为源于自然的手性配体参与的催 化反应而言，仍然具有重大的理论意义.

Ricci 等 ${ }^{[26]}$ 合成了壳聚糖气凝胶, 可用于水相中的 不对称醇醛缩合反应(Eq. 1). 其催化对硝基苯甲醛和环 己酮的缩合反应，缩合产物的分离产率及 $a n t i$ 构型产物 的 $e e$ 值最高分别达到 $88 \%$ 、93\%. 当反应体系中加入

* E-mail: wangjiaxi@hebut.edu.cn

Received June 16, 2014; revised July 18, 2014; published online September 12, 2014.

Project supported by the Natural Science Foundation of Hebei Province (No. B2011202087).

河北省自然科学基金(No. B2011202087)资助项目. 


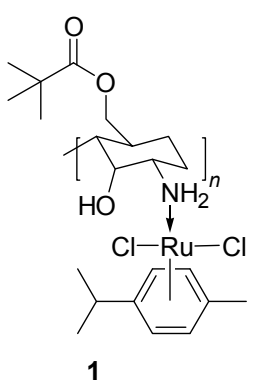

2,4-二硝基苯酚或硬脂酸时, 产率能够明显提高. 催化 剂在循环使用四次后, 依然保持较高的催化活性(产率 $87 \%$ ) 和对映体选择性 (anti 构型产物的 $83 \% e e$ ). Cui 等 ${ }^{[27]}$ 首次发现壳聚糖负载 $L$-脯氨酸催化剂可催化多种芳香 醛与硝基甲烷的不对称 Henry 反应, 水可以溶胀壳聚糖 负载 $L$-脯氨酸形成水溶胶束, 作为一种非均相催化剂, 该溶胶束体系具有出较高的循环使用性能.

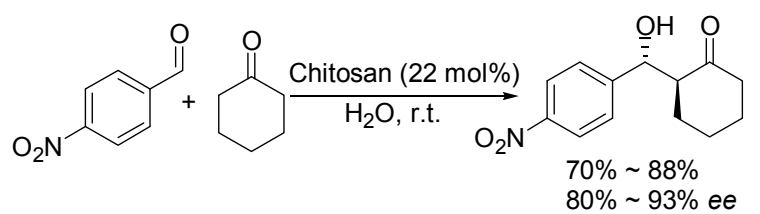

Crucianelli 等 ${ }^{[34]}$ 制备出一系列修饰甲壳素、壳聚糖 负载甲基三氧化铼催化剂 2 . 在催化烯烃环氧化反应中, 发现催化剂 2 有很好的催化活性, 将催化剂 2 负载到二 氧化硅上, 催化活性得到进一步提高. 其催化反式 $\beta$-甲 基苯乙烯环氧化的转化率最高 $98 \%$, 产物的 ee 值为 $98 \%$.

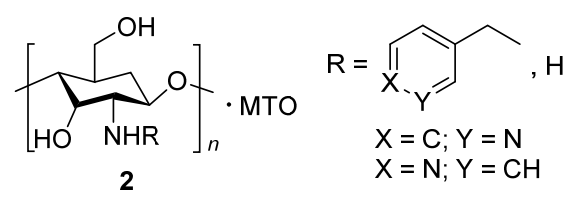

\section{2 烯丙基取代反应}

过渡金属催化烯丙基取代反应在有机合成中具有 重要的学术意义. 该催化反应具有通用性强、条件温和、 区域和立体选择性多变等特点. Dez 等 ${ }^{[35,36]}$ 制备了壳聚 糖负载离子液体与 Pd 配合物一起催化 Tsuji-Trost 烯丙 基取代反应(Eq. 2). 催化剂优势在于其具有广泛的适用 性, 催化剂用量小, 分离方便. 当以三磺化三苯基膦 (TPPTS) 作为钯离子的配体时, 其循环使用次数可达 10 次以上.

\section{3 偶联反应}

碳一碳键生成的偶联反应是重要的化学反应之一, 利用这些反应可以将简单的反应前体转变为结构复杂 的分子 ${ }^{[37]}$. 壳聚糖及其衍生物金属配合物作为偶联反

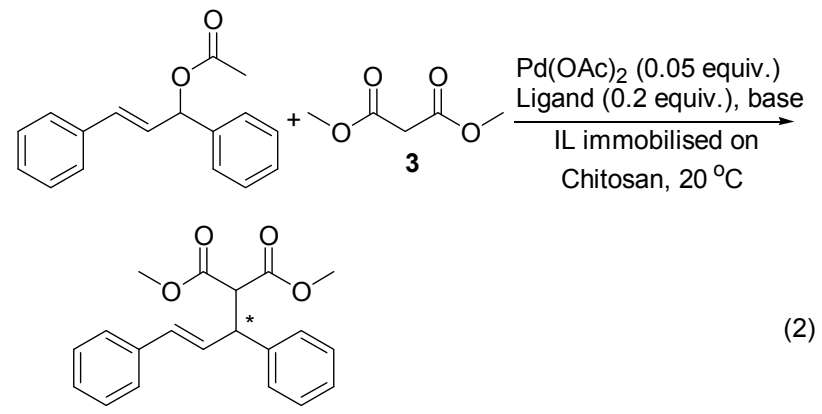

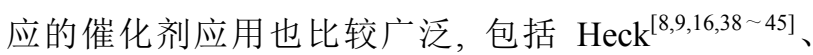

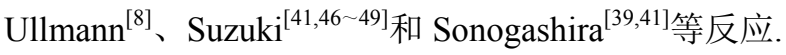

Calo 等 ${ }^{[50]}$ 利用壳聚糖负载 Pd 催化剂催化卤代苯与 丙烯酸丁酯的 Heck 反应(Eq. 3), 反应以四丁基溴化铵 (TBAB)为溶剂, 四丁基乙酸铵(TBAA)为碱, 催化溴苯 和丙烯酸正丁酯偶联反应，由于形成高活性的 Pd 胶体 能够稳定存在于反应溶剂中, 在碱的作用下能够快速形 成 Pd-H 的活性中间体，反应在 $15 \mathrm{~min}$ 内即可达到 $99 \%$ 的转化率.

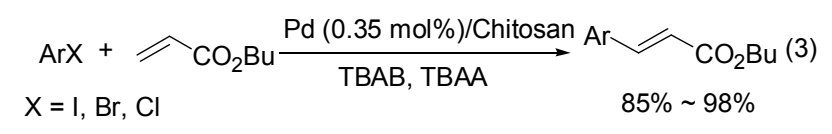

Cravotto 等 ${ }^{[47]}$ 通过将壳聚糖、 $\mathrm{Pd}(\mathrm{OAc})_{2} 、 4,4-$ 二环 己基甲烷二异氰酸酯(HMDI)共混制备了 HMDI 交联壳 聚糖负载 Pd 催化剂, 此催化剂可用于卤代芳烃 Suzuki 偶联反应(Eq. 4), 并获得较好的产率. Lee 等 ${ }^{[49]}$ 制备了壳 聚糖-均三嗪-甲基聚乙二醇和甲基聚乙二醇 Pd 催化剂, 在没有相转移试剂的条件下, 能够高效催化 Suzuki 反 应，当以 CS-g-mPEG350 Pd(0)为催化剂、 $\mathrm{Pd}$ 用量为 0.5 mol $\%$ 时, 联苯的产率达 $92 \%$.

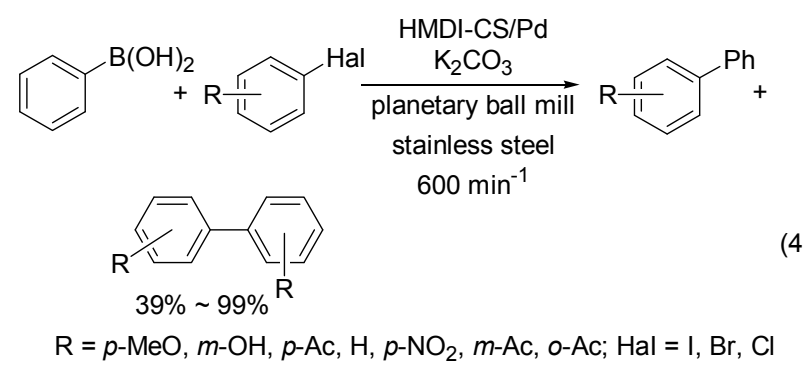

Quignard 等 ${ }^{[51]}$ 通过超临界 $\mathrm{CO}_{2}$ 干燥制备壳聚糖纤 维负载纳米 $\mathrm{Au}$ 催化 Sonogashira 反应(Eq. 5)及芳基硼酸 的自身偶联反应，通过 XPS 分析，催化剂中金负载量为 $0.5 \mathrm{wt} \%\left(\mathrm{Au}^{0}\right.$ 含量为 $70.3 \% 、 \mathrm{Au}^{3+}$ 为 $16.4 \% 、 \mathrm{Au}^{+}$为 $13.2 \%$ ), 其中 $\mathrm{Au}^{+}$与 $\mathrm{Au}^{3+}$ 为催化 Sonogashira 反应和芳 基嗍酸自偶联反应的活性物种. 通过控制纳米金在壳聚 糖表面的增长, 使其能够形成具有催化活性的物种, 从 而高效催化 Sonogashira 反应. 


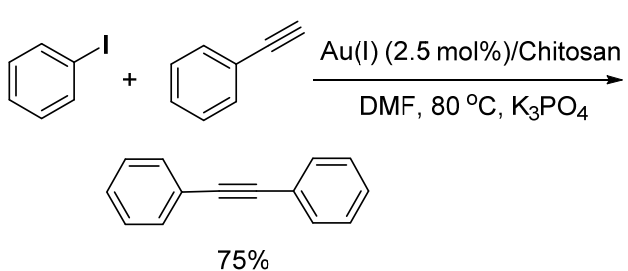

(5)

Sakurai 等 ${ }^{[52]}$ 以硼氢化钠为还原剂, 壳聚糖作为载 体, 通过共还原法制备了一系列的壳聚糖负载 $\mathrm{Au} / \mathrm{Pd}$ 纳 米簇催化剂催化芳基硼酸的自身氧化偶联反应(Eq. 6), 其中当 $\mathrm{Au} / \mathrm{Pd}$ 为 $0.81 / 0.19$ 时, 催化剂的催化活性最高, 甲基苯硼酸偶联生成对甲基联苯 4 的产率为 $99 \%$.

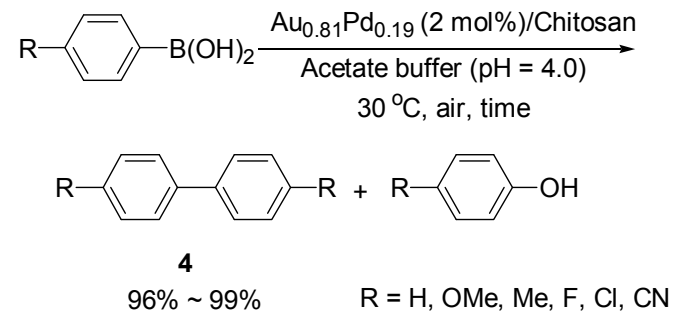

Alesi 等 ${ }^{[53]}$ 利用壳聚糖负载 Pd 为催化剂, 在微波条 件下催化低聚噻吩的偶合反应, 此反应比传统均相催化 反应更加绿色高效, 当以碘代噻吩为底物, $\mathrm{KF}$ 为碱, 反 应 30 100 min, 产率可达 87\%.

\section{4 加成反应}

Sabater 等 ${ }^{[54]}$ 制备了壳聚糖负载二氧化硅 $\mathrm{Au}$ 配合物 催化剂用于炔烃的选择性氢胺化反应(Scheme 1). 以 1辛炔为底物, 在 $100{ }^{\circ} \mathrm{C}$ 下反应 $22 \mathrm{~h}$, 底物转化率为 $100 \%$, 酮与亚胺的选择性分别为 $36 \%$ 和 $64 \%$. 在反应过 程中, 催化剂未被溶解, 能够充分的保证催化剂的活性 和使用寿命.

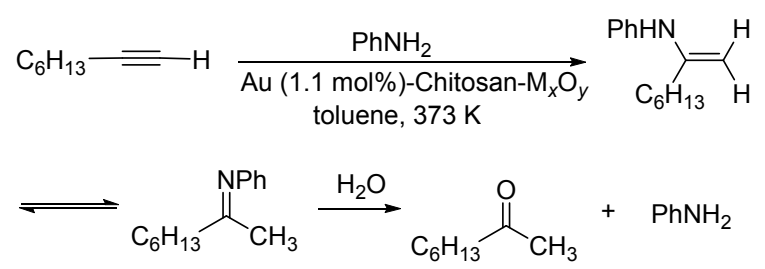

图式 1 炔烃的氢胺化反应

Scheme 1 Hydroamination reactions of alkynes

2002 年, Sharpless 等 ${ }^{[55]}$ 报道了 $\mathrm{Cu}(\mathrm{I})$ 催化叠氮化合 物与末端炔烃的环加成反应. $\mathrm{Cu}(\mathrm{I})$ 催化剂的存在使该类 环加成反应不仅能够在温和条件下进行, 而且具有极高 的区域选择性和产率, 被视为“点击化学” 的典范. Taran 等 ${ }^{[56]}$ 首次制备了一系列的壳聚糖-席夫碱 $\mathrm{Cu}(\mathrm{I})$ 配合物 催化剂催化叠氮化合物环加成反应(Eq. 7), 获得了比较 好的效果. 当选择配体 5 时，催化剂用量 $0.1 \mathrm{~mol} \%$, $\mathrm{EtOH}$ 和 $\mathrm{H}_{2} \mathrm{O}$ 作为溶剂, 6 的产率为 $99 \%$.<smiles>O=Cc1c([C@H](C=O)OCc2ccccc2)ccc2ccc(C=NOc3ccccc3)nc12</smiles>

Strecker 反应是制备氨基酸类化合物的重要反应. Dekamin 等 ${ }^{[57]}$ 利用商业壳聚糖为催化剂, 当壳聚糖用量 为 $12 \mathrm{mg}$, 对氯苯甲醛 $1 \mathrm{mmol}$, 苯胺 $1 \mathrm{mmol}$, 三甲基氰 基硅(TMSCN)为底物的 1.2 倍， 7 的产率可达 98\% (Eq. $8)$.

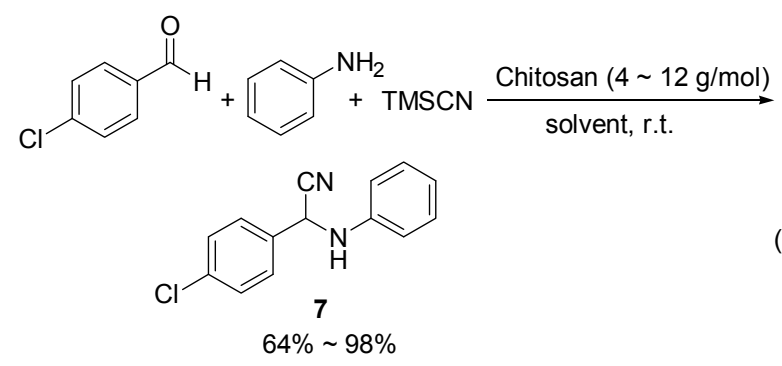

El Kadib 等 ${ }^{[58]}$ 制备了壳聚糖负载二氧化钛水凝胶催 化剂，并用于催化 Henry (Eq. 9)、Michael 加成反应(Eq. 10), Knoevenagel 反应(Eq. 11), 催化剂具有良好的催化 性能, 当以 $\mathrm{Ti}(\mathrm{Acac})_{2}(\mathrm{O} i-\mathrm{Pr})_{2}$ 为前体合成的壳聚糖 (a) $\mathrm{TiO}_{2}$ 作为催化剂, 8 10 的产率分别为 97\%、97\%、 $86 \%$. Shukla 等 ${ }^{[59]}$ 将壳聚糖进行简单处理，制成水凝胶， 经干燥粉碎后直接用于催化反应合成茉莉醛 $\mathbf{1 0}$, 反应 在 $160{ }^{\circ} \mathrm{C}$ 的无溶剂下进行，底物的转化率大于 $99 \%$, 茉 莉醛的收率可达 $88 \%$.

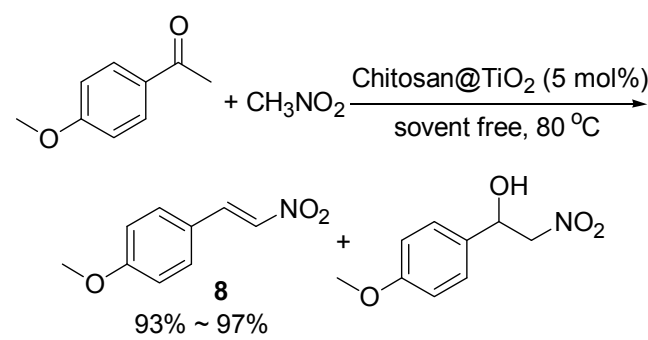<smiles>C=C(C=C=[C+]C(C)=O)C(=O)OCC</smiles>

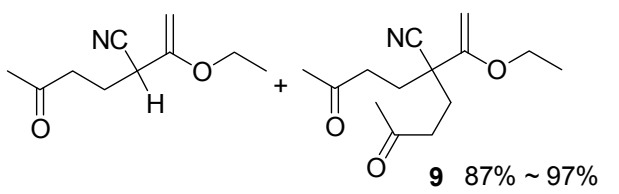




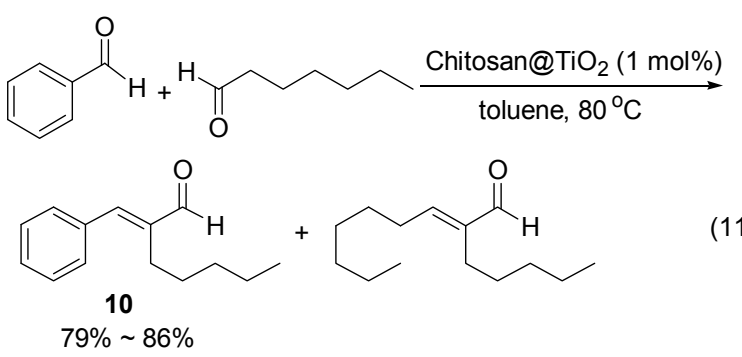

Shan 等 ${ }^{[00]}$ 通过一步法制备杂多酸/壳聚糖负载磁性 纳米 $\mathrm{Fe}_{3} \mathrm{O}_{4}$ 催化剂, 交联壳聚糖可以保护纳米粒子不被 杂多酸和其他酸性环境的侵蚀, 并能够针固杂多酸, 提 高催化剂的催化性能, 此催化剂可用于苯甲醛和乙二醇

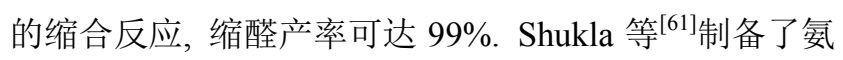
丙基三甲氧基硅烷修饰壳聚糖催化剂, 用于 $\mathrm{C}_{3} \sim \mathrm{C}_{7}$ 的 醛类的羟醛缩合反应, 能够生成 $\alpha, \beta$-不饱和醛类. 当催 化剂/乙醛用量为 $100 \mathrm{mg} / \mathrm{g}$ 时, 反应 $2 \mathrm{~h}$ 就能实现底物的 $100 \%$ 转化, 动力学显示, 反应速率随着戊醛、催化剂 量、温度的增加而增加, 壳聚糖催化剂的加入避免了酸 和碱的使用。

\section{5 氧化反应}

环己酮和环己醇是重要的有机化工原料. 壳聚糖负 载金属配合物催化剂催化环己烷氧化制备环己酮和环 己醇的工作已有报道, 已报道的催化剂有壳聚糖和改性 壳聚糖负载的 $\mathrm{Fe} 、 \mathrm{Cu} 、 \mathrm{Co} 、 \mathrm{Ni} 、 \mathrm{Au} 、 \mathrm{Pd}$ 等配合物 ${ }^{[11 ~ 15,62]}$. Guo 等 ${ }^{[63]}$ 制备了壳聚糖负载卟啉 $\mathrm{Fe}(\mathrm{III})$, 在无溶剂条件 下催化环己烷的氧化, 结果发现壳聚糖负载的催化剂比 单纯的卟啉 $\mathrm{Fe}(\mathrm{III})$ 的催化活性要高. 在 $418 \mathrm{~K}, 0.8 \mathrm{MPa}$ 条件下, 环已烷的转化率为 $10.48 \%$, 环己酮与环己醇的 选择性为 $79.2 \%$. 其转换频率是单独卟啉铁的 22 倍.

Labhsetwar 等 ${ }^{[64]}$ 制备了壳聚糖负载介孔 $\mathrm{TiO}_{2}$ 、 $\mathrm{ZrO}_{2} 、 \mathrm{Al}_{2} \mathrm{O}_{3}$ 并负载 $\mathrm{Cu}-\mathrm{Mn}$ 催化剂, 用于甲苯和乙醛的 催化氧化, 催化活性顺序为 $\mathrm{Cu}-\mathrm{Mn} / \mathrm{TiO}_{2}>\mathrm{Cu}-\mathrm{Mn} /$ $\mathrm{ZrO} \mathrm{O}_{2}>\mathrm{Cu}-\mathrm{Mn} / \mathrm{Al}_{2} \mathrm{O}_{3}$. 其中 $\mathrm{Cu}-\mathrm{Mn} / \mathrm{TiO}_{2}$ 和 $\mathrm{Cu}-\mathrm{Mn} / \mathrm{ZrO}{ }_{2}$ 在 $350{ }^{\circ} \mathrm{C}$ 实现甲苯的完全转化, 而 $\mathrm{Cu}-\mathrm{Mn} / \mathrm{Al}_{2} \mathrm{O}_{3}$ 在 $400{ }^{\circ} \mathrm{C}$ 时实现完全转化; 当以乙醛为底物, 在 $80{ }^{\circ} \mathrm{C}$ 时, 以 $\mathrm{Cu}-\mathrm{Mn} / \mathrm{TiO}_{2}, \mathrm{Cu}-\mathrm{Mn} / \mathrm{ZrO}_{2}, \mathrm{Cu}-\mathrm{Mn} / \mathrm{Al}_{2} \mathrm{O}_{3}$ 为催化剂的 转化率分别为 $45 \%$ 、20\%、5\%. Huang 等 ${ }^{[65,66]}$ 利用壳聚 糖负载四苯基卟啉 $\mathrm{Mn}(\mathrm{III})$, 与四苯基卟啉 $\mathrm{Mn}(\mathrm{III})$ 进行 对比, 前者对甲苯氧化的催化活性更强, 甲苯转化率为 $5.9 \%$, 苯甲醛和苯甲醇的选择性为 $96 \%$, 当用 $\mathrm{Co}$ (II)作 催化剂时, 甲苯的转化率为 $8.83 \%$, 苯甲醛和苯甲醇的 选择性为 $65 \%$. Hall 等 ${ }^{[67]}$ 用壳聚糖负载 $\mathrm{Au} / \mathrm{Pd}$ 合金, 通 过熔融和不可控的纳米颗粒的增长, 制备出粒径为 3 纳 米的催化剂颗粒, 并用此催化剂催化苯甲醇类化合物氧 化成为苯甲醛. 当底物为间氯代苯甲醇时, 其 TON 值为
$10 \mathrm{~h}^{-1}$ ，间氯代苯甲醛的产率为 $97 \%$.

$\mathrm{Li}$ 等 ${ }^{[68]}$ 通过固相法制备出壳聚糖负载树状锡配合 物催化剂 11, 用于酮类的 Baeyer-Villiger 氧化重排反应 (Eq. 12). 该催化剂在双氧水的存在下催化金刚烷酮的 Baeyer-Villiger 氧化反应最高转化率可达 $99 \%$, 选择性 $100 \%$. 由于催化剂不溶于有机溶剂, 可以多次回收利 用.
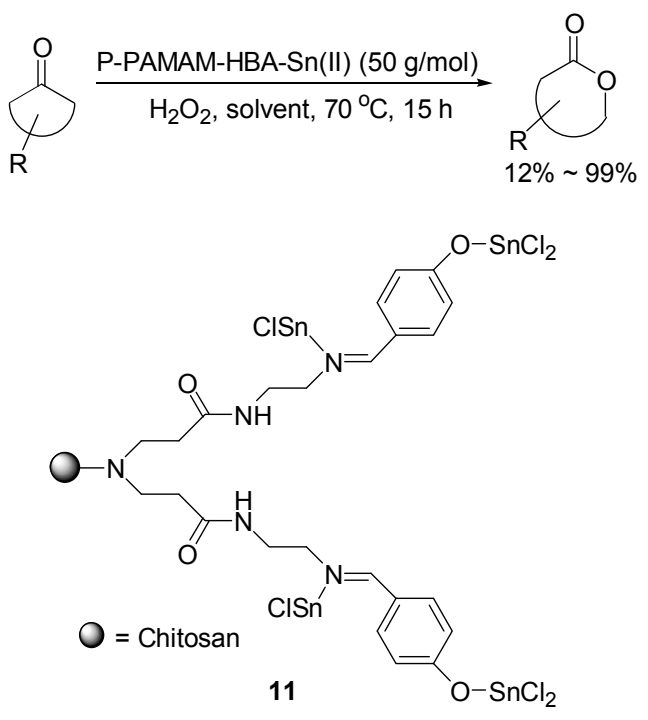

\section{6 还原反应}

Gong 等 ${ }^{[23]}$ 制备了 $\mathrm{SiO}_{2}$-CS-Schiff 基 Pd 催化剂, 用 于苯乙酮的催化还原, 相比于 $\mathrm{PdCl}_{2}$ 和 $\mathrm{SiO}_{2}-\mathrm{CS}-\mathrm{Pd}$, CS-Schiff-Pd 和 $\mathrm{SiO}_{2}$-CS-Schiff-Pd 有着更强的催化活性, 能够将苯乙酮直接还原成乙苯. 当以乙醇为溶剂、氢气 流速 $45 \mathrm{~mL} / \mathrm{min} \mathrm{H}_{2}$, 温度为 $313 \mathrm{~K}$ 时, 乙苯的产率可以 达到 $100 \%$. Bajaj 等 ${ }^{[69]}$ 制备了 $N$-修饰壳聚糖负载 Pd 催 化剂, 并催化还原苯基环氧乙烷, 其中 $N$-修饰氨壳聚糖 基 $\mathrm{Pd}$ 配合物作为催化剂时, 2-苯乙醇的产率为 $85 \%$. Ondruschka 等 ${ }^{[70]}$ 制备了六亚甲基二异氧酸酯修饰壳聚 糖基 $\operatorname{Pd}(\mathrm{II})$ 配合物，并催化环己烯二酮、查尔酮、柠檬 醛、1,2-二苯乙炔、 $N$-茮烯苯胺等各种烯烃及 $\alpha, \beta$-不饱 和醛酮的加氢, 其对双键的选择性能够达到 $99 \%$.

壳聚糖负载的 $\mathrm{Ag}$ 和 $\mathrm{Au}^{[71,72]}$ 可以催化对硝基苯酚的 还原(Eq. 13). 纳米金粒子在壳聚糖载体上稳定存在, 循 环使用 11 次之后并未发现催化剂失活迹象. Chattopadhyay 等 ${ }^{[73]}$ 制备壳聚糖纳米 $\mathrm{Ag}$ 复合物催化剂, 此催 化剂可用于催化还原 4-硝基苯酚, 并且催化剂重复利用 10 次后依然保持很高的催化活性.

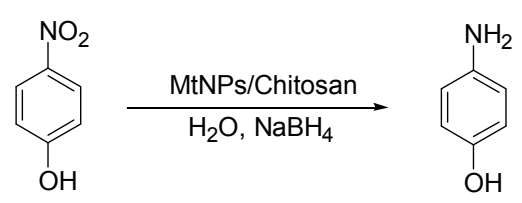




\section{7 其他反应}

\section{1 氢甲酰化反应}

烯烃的氢甲酰化反应是一类重要的均相催化反应, 有着广泛的工业前景, 对于农药、香料、药物以及天然 产物合成方面具有重要意义 ${ }^{[74]}$. Smith 等 ${ }^{[75]}$ 合成了壳聚 糖席夫碱 $\mathrm{Rh}(\mathrm{I})$ 配合物催化剂 12, 催化 1-辛烯的加氢甲 酰化反应(Eq. 14). 催化 1-辛烯生成醛类化合物的最佳 反应温度为 $75{ }^{\circ} \mathrm{C}$, 混合气压 $3.0 \mathrm{MPa}$, 形成 $52 \% \sim 95 \%$ 的醛类和一部分异辛稀. 这类催化剂相比于 $\left[\mathrm{Rh}(\mathrm{CO})_{2}-\right.$ $(\mathrm{acac})_{2}$ ] 催化剂有着相似的活性和更高的选择性, 在循 环使用四次后，催化反应仍然能够获得 $75 \%$ 79\%的转 化率.
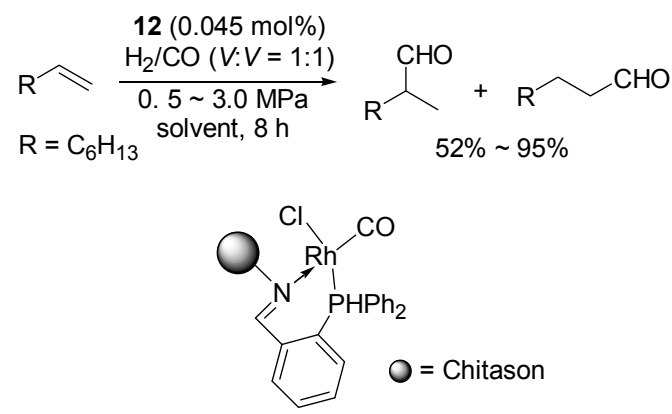

12

\section{2 一氧化碳和二氧化碳的插入反应}

$\operatorname{Park}^{[76]}$ 和 $\mathrm{He}$ 等 ${ }^{[21]}$ 制备了壳聚糖-季铵盐化合物 (QCHT) 13 用于催化环氧化合物与二氧化碳反应合成碳 酸酯类化合物(Eq. 15), 当 $\mathrm{R}=\mathrm{Me}, \mathrm{X}=\mathrm{I}$ 时, 转化率= $100 \%$, 碳酸酯的产率大于 $99 \%$. 其催化机理可能是因为 壳聚糖中的 $\mathrm{OH}$ 与环氧化合物形成氢键, 促使卤素负离 子对环氧化合物 $\beta$ 碳原子的加成, 形成了开环中间体, 然后 $\mathrm{CO}_{2}$ 进一步加成, 形成环碳酸脂类化合物. Zhang 等 ${ }^{[77]}$ 制备了壳聚糖-1-乙基-2-甲基咪唑嗡盐卤代物配合 物 14 , 催化环氧化合物与二氧化碳反应合成碳酸酯类 化合物，该反应在微波下进行，转化率最高为 $95 \%{ }^{[78]}$.



羰基插入反应是有机化学乃至工业应用中比较重 要的反应，通过对 $\mathrm{C}-\mathrm{X}$ ( $\mathrm{X}=\mathrm{Cl}, \mathrm{Br}$ 等)的羰基插入反应 可以合成一系列羰基化合物，符合 “原子经济” 和 “绿 色化学” 的要求 ${ }^{[79] . X i a ~}$ 等 $^{[80]}$ 利用壳聚糖制备了壳聚糖一 席夫碱-Co(II)配合物在碘化钠的作用下催化 2-氨基醇的 羰基化反应(Eq. 16), 并获得了较高的产率. 当 $\mathrm{R}^{1}=$ $\mathrm{R}^{3}=\mathrm{H}, \mathrm{R}^{2}=\mathrm{Me}$ 时, 2-氨基醇转化率为 $100 \%, 15$ 的选择 性为 $98 \%$.

$$
\begin{aligned}
& \mathrm{R}^{1}=\mathrm{H}, \mathrm{Me}, \mathrm{Et}, \mathrm{i}-\mathrm{Pr}, \mathrm{Me}_{2} ; \mathrm{R}^{2}=\mathrm{H}, \mathrm{Me} ; \\
& \mathrm{R}^{3}=\mathrm{H}, \mathrm{CH}_{2} \mathrm{CH}_{2} \mathrm{OH}
\end{aligned}
$$

\section{3 水解反应}

Xiang 等 ${ }^{[81]}$ 制备了 $N-16$ 烷基- $O$-硫酸盐修饰壳聚糖 负载 $\mathrm{Cu}(\mathrm{II})$, 用于非均相催化磷酸酯(BNPP)的水解反应, 其催化水解速率是非催化 BNPP 水解的 $7 \times 10^{4}$ 倍. 其水 解机理可能是, $\mathrm{Cu}(\mathrm{II})$ 为路易斯酸, 能够作为亲电试剂破 坏磷酸酯键 16. Chen 等 ${ }^{[82]}$ 制备了 $\mathrm{Cu}(\mathrm{II})$ 壳聚糖磁性纳米 粒子催化剂用于 BNPP 的水解反应，催化剂在 $\mathrm{p} K_{\mathrm{a}}$ 值 12 时催化水解速率最低, 虽然相比于其他催化剂 $\mathrm{Cu}(\mathrm{II})$ 壳 聚糖磁性纳米粒子对 BNPP 的亲和力较低, 但其催化速 率常数 $k_{\mathrm{cat}}$ 和最大反应常数 $V_{\max }$ 却高于其他文献记录水

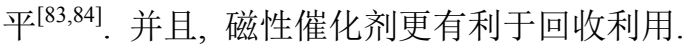

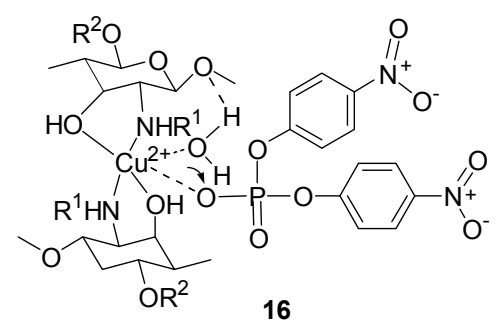

\section{8 结论}

壳聚糖是一种天然高分子化合物, 含有大量的氨基 和羟基，易于修饰及与金属离子配位，形成功能壳聚糖 衍生物及壳聚糖负载的金属催化剂. 壳聚糖分子上的氨 基作为有机碱催化剂的研究也引起人们的关注，其功能 化修饰有望成为新一代有机碱催化剂. 壳聚糖及其衍生 物分子上的手性中心对壳聚糖及其负载金属催化剂在 不对称催化反应具有重要的影响. 壳聚糖及其衍生物溶 解性可调, 同金属络合后形成的催化剂具有较好的稳定 性, 方便分离. 作为一种低成本、环境友好型催化剂及 催化剂载体, 壳聚糖及其功能衍生物在非均相催化工业 中的应用也是未来研究的一个重要方向. 


\section{References}

[1] Guibal, E.; Vincent, T.; Touraud, E.; Colombo, S.; Ferguson, A. J. Appl. Polym. Sci. 2006, 100, 3034.

[2] Kuehbeck. D.; Saidulu, G.; Redd, K. R.; Diaz, D. Green Chem. 2012, 14, 378.

[3] Reddy, K. R.; Rajgopal, K.; Maheswari, C. U.; Kantam, M. L. New J. Chem. 2006, 30, 1549.

[4] Zhang, J.-T.; Wei, G.; Keller, T. F.; Gallagher, H.; Stoetzel, C.; Mueller, F. A.; Gottschaldt, M.; Schubert, U. S.; Jandt, K. D. Macromol. Mater. Eng. 2010, 295, 1049.

[5] Primo, A.; Atienzar, P.; Sanchez, E.; Delgado, J. M.; Garcia, H. Chem. Commun. 2012, 48, 9254 .

[6] El Kadib, A.; Bousmina, M. Chem-Eur. J. 2012, 18, 8264.

[7] Zeng, M.; Zhang, X.; Shao, L.; Qi, C.; Zhang, X.-M. J. Org. Chem. 2012, 704, 29.

[8] Zeng, M.; Zhang, X.; Qi, C.; Zhang, X.-M. Int. J. Biol. Macromol. 2012, 51,730

[9] Dhar, J.; Patil, S. ACS Appl. Mater. Inter. 2012, 4, 1803.

[10] Zhai, H.; Zhang, A.; Li, L.; Long, S. J. Appl. Polym. Sci. 2012, 123, 2140 .

[11] Xiong, Y.; Wang, H.; Wu, C.; Wang, R. Polym. Adv. Technol. 2012, 23, 1429.

[12] Tsvetkova, I. B.; Bronstein, L. M.; Sidorov, S. N.; Lependina, O. L.; Sulman, M. G.; Valetsky, P. M.; Stein. B.; Nikoshvili, L. Z.; Matveeva, V. G.; Sidorov, A. I.; Tikhonov, B. B.; Demidenko, G. N.; Kiwi-Minsker, L.; Sulman, E. M. J. Mol. Catal. A: Chem. 2007, 276, 116.

[13] Yu, J.; Liu, T. In Eco-Materials Processing and Design X, Eds.: Kim, H.; Yang, J. F.; Sekino, T.; Lee, S. W., ISEPD, Xian, China, 2009, p. 537.

[14] Peirano, F.; Vincent, T.; Quignard, F.; Robitzer, M.; Guibal, E. $J$. Membr. Sci. 2009, 329, 30.

[15] Bradshaw, M.; Zou, J.; Byrne, L.; Iyer, K. S.; Stewart, S. G.; Raston, C. L. Chem. Commun. 2011, 47, 12292.

[16] Schiffman, J. D.; Schauer, C. L. Biomacromolecules 2007, 8, 2665.

[17] Chang, Y.-C.; Chen, D.-H. J. Colloid Interface Sci. 2005, 283, 446.

[18] Chang, Y.-C.; Chang, S.-W.; Chen, D.-H. React. Funct. Polym. 2006, 66, 335.

[19] Falco, M.; Retuert, J.; Hidrobo, A.; Covarrubias, C.; Araya, P.; Sedran, U. Appl. Catal. A 2009, 366, 269.

[20] Demetgül, C. Carbohydr. Polym. 2012, 89, 354.

[21] Zhao, Y.; Tian, H.-S.; Qi, X.-H.; Han, Z.-N.; Zhuang, Y.-Y.; He, L.-N. J. Mol. Catal. A: Chem. 2007, 271, 284.

[22] Sun, C.; Hu, B.; Zhao, D.; Liu, Z. J. Appl. Polym. Sci. 2012, 125, 79.

[23] Gong, S.-W.; He, H.-F.; Zhao, C.-Q.; Liu, L.-J.; Cui, Q.-X. Synth. Commun. 2012, 42, 574.

[24] Guibal, E. Prog. Polym. Sci. 2005, 30, 71.

[25] Macquarrie, D. J.; Hardy, J. J. E. Ind. Eng. Chem. Res. 2005, 44, 8499.

[26] Ricci, A.; Bernardi, L.; Gioia, C.; Vierucci, S.; Robitzer, M.; Quignard, F. Chem. Commun. 2010, 46, 6288.

[27] Cui, Y.-C.; Zhang, H.-F.; Li, R.-T.; Liu, Y.; Xu, C. Chin. J. Org. Chem. 2010, 30, 707 (in Chinese)

(崔元臣, 张和风, 李润涛, 刘毅, 许楚, 有机化学, 2010, 30, 707.)

[28] Babin, M.; Clement, R.; Gagnon, J.; Fontaine, F. G. New J. Chem. 2012, 36, 1548 .

[29] Zhang, H.; Zhao, W.; Zou, J.; Liu, Y.; Li, R.; Cui, Y. Chirality 2009, 21,492 .

[30] Mac Leod, T. C. O.; Palaretti, V.; Barros, V. P.; Faria, A. L.; Silva,
T. A.; Assis, M. D. Appl. Catal. A 2009, 361, 152.

[31] Qin, Y.; Zhao, W.; Yang, L.; Zhang, X.; Cui, Y. Chirality 2012, 24, 640.

[32] Zhang, H.-F.; Zhang. L.; Cui, Y.-C. React. Funct. Polym. 2007, 67, 322.

[33] Gioia, C.; Ricci, A.; Bernardi, L.; Bourahla, K.; Tanchoux, N.; Robitzer, M.; Quignard, F. Eur. J. Org. Chem. 2013, 3, 588.

[34] Giuseppe, A. D.; Crucianelli, M.; Passacantando, M.; Nisi, S.; Saladino, R. J. Catal. 2010, 276, 412.

[35] Baudoux, J.; Perrigaud, K.; Madec, P. J.; Gaumont, A. C.; Dez, I. Green Chem. 2007, 9, 1346.

[36] Moucel, R.; Perrigaud, K.; Goupil, J. M.; Madec, P. J.; Marinel, S.; Guibal, E.; Gaumont, A. C.; Dez, I. Adv. Synth. Catal. 2010, 352, 433.

[37] Zhang, Y.; Luo, S.; Zhu, C.-J. Chin. J. Org. Chem. 2012, 32, 2073 (in Chinese). (张艳，罗莎，朱成建，有机化学, 2012, 32, 2073.)

[38] Yang, Y.; Li, G.; Song, Z.; Yang, X.; Liu, P. Lett. Org. Chem. 2010, 7, 533.

[39] Leonhardt, S. E. S.; Ondruschka, B.; Cravotto, G.; Leo, C. D.; Jandt, K. D.; Keller, T. F. Appl. Catal. A 2010, 379, 30.

[40] Xu, X.; Liu, P.; Li, S.-H.; Zhang, P.; Wang, X.-Y. React. Kinet. Catal. Lett. 2006, 88, 217.

[41] Gronnow, M. J.; Luque, R.; Macquarrie, D. J.; Clark, J. H. Green Chem. 2005, 7, 552 .

[42] Cui, Y.-C.; Zhang, L.; Li, Y. Polym. Adv. Technol. 2005, 16, 633.

[43] Liu, P.; Liu, Y.; Li, J.-Y.; Liu, Y.-Z.; Lu, W.-P.; Wang, X.-Y.; Li, L.-M. Chin. J. Org. Chem. 2007, 27, 87 (in Chinese).

(刘蒲, 刘晔, 李晶玉, 刘一真, 卢伟鹏, 王向宇, 李利民，有机 化学, 2007, 27, 87.)

[44] Chen, J.; Tang, J.-J.; Hu, C.-H.; Wang, J.-T. Chin. J. Inorg. Chem. 2009, 25, 147 (in Chinese). (陈静，唐俊杰，胡晨晖，王锦堂，无机化学学报，2009, 25, 147.)

[45] Li, X.-W.; Zi, W.-W.; Guo, S.-W.; Xiao, D.-L.; Jian, G.-R. Polym. Adv. Technol. 2010, 21, 244.

[46] Cotugnoa, P.; Casielloa, M.; Naccia, A.; Mastrorillic, P.; Dell'Annac, M. M.; Monopolia, A. J. Organomet. Chem. 2014, 752, 1.

[47] Cravotto, G.; Garella, D.; Tagliapietra, S.; Stolle, A.; Schuessler, S.; Leonhardt, S. E. S.; Ondruschka, B. New J. Chem. 2012, 36, 1304.

[48] Lasri, J.; Mac Leod, T. C. O.; Pombeiro, A. J. L. Appl. Catal. A 2011, 397, 94

[49] Sin, E.; Yi, S.-S.; Lee, Y.-S. J. Mol. Catal. A: Chem. 2010, 315, 99.

[50] Calo, V.; Nacci, A.; Monopoli, A.; Fornaro, A.; Sabbatini, L.; Cioffi, N.; Ditaranto, N. Organometallics 2004, 23, 5154.

[51] Primo, A.; Quignard, F. Chem. Commun. 2010, 46, 5593.

[52] Sophiphun, O.; Wittayakun, J.; Dhital, R. N.; Haesuwannakij, S.; Murugadoss, A.; Sakurai, H. Aust. J. Chem. 2012, 65, 1238.

[53] Alesi, S.; Di Maria, F.; Melucci, M.; Macquarrie, D. J.; Luque, R.; Barbarella, G. Green Chem. 2008, 10, 517.

[54] Corma, A.; Concepcion, P.; Dominguez, I.; Fornes, V.; Sabater, M. J. J. Catal. 2007, 251, 39.

[55] Rostovtsev, V. V.; Green, L. G.; Fokin, V. V.; Sharpless, K. B. Angew. Chem., Int. Ed. 2002, 114. 2708.

[56] Chtchigrovsky, M.; Primo, A.; Gonzalez, P.; Molvinger, K.; Robitzer, M.; Quignard, F.; Taran, F. Angew. Chem., Int. Ed. 2009, 48, 5916.

[57] Dekamin, M. G.; Azimoshan, M.; Ramezani, L. Green Chem. 2013, 15,811 .

[58] El Kadib, A.; Molvinger, K.; Bousmina, M.; Brunel, D. Org. Lett. 2010, 12, 948.

[59] Sudheesh, N.; Sharma, S. K.; Shukla, R. S. J. Mol. Catal. A: Chem. 2010, 321, 77 . 
[60] Kong, A.; Wang, P.; Zhang, H.; Yang, F.; Huang, S.; Shan, Y. Appl. Catal. A 2012, 417, 1839.

[61] Jose, T.; Sudheesh, N.; Shukla, R. S. J. Mol. Catal. A: Chem. 2010, $333,158$.

[62] Sun, C.; Hu, B.; Zhao, D.; Liu, Z. J. Appl. Polym. Sci. 2012, 125, 79.

[63] Guo, C.-C.; Huang, G.; Zhang, X.-B. Appl. Catal. A: Gen. 2003, 247, 261.

[64] Doggali, P.; Teraoka, Y.; Mungse, P.; Shah, I. K.; Rayalu, S.; Labhsetwar, N. J. Mol. Catal. A: Chem. 2012, 358, 23.

[65] Huang, G.; Guo, Y.-A.; Zhou, H.; Zhao, S.-K.; Liu, S.-Y.; Wang, A.-P.; Wei, J.-F. J. Mol. Catal. A: Chem. 2007, 273, 144.

[66] Huang, G.; Wang, A.-P.; Liu, S.-Y.; Guo, Y.-A.; Zhou, H.; Zhao, S.-K. Catal. Lett. 2007, 114, 174.

[67] Hall, S. R.; Collins, A. M.; Wood, N. J.; Ogasawara, W.; Morad, M.; Miedziak, P. J. RSC Adv. 2012, 2, 2217.

[68] Li, C.; Lei, Z.; Ma, H.; Wu, S.; Sun, Q. J. Dispersion Sci. Technol. 2012, 33, 983 .

[69] Dabbawala, A. A.; Sudheesh, N.; Bajaj, H. C. Dalton. Trans. 2012, 41, 2910.

[70] Schüßler, S.; Blaubach, N.; Stolle, A.; Cravotto, G.; Ondruschka, B. Appl. Catal. A. 2012, 445, 231

[71] Wei, D.; Ye, Y.; Jia, X.; Yuan, C.; Qian, W. Carbohydr. Res. 2010, 345,74 .
[72] Chang, Y.-C.; Chen, D.-H. J. Hazard. Mater. 2009, 165, 664.

[73] Murugadoss, A.; Chattopadhyay, A. Nanotechnology 2008, 19, 1.

[74] Jia, X.-F.; Wang, Z.; Xia, C.-G.; Ding, K.-L. Chin. J. Org. Chem. 2013, 33, 1369 (in Chinese).

(贾肖飞, 王正, 夏春谷, 丁奎岭, 有机化学, 2013, 33, 1369.)

[75] Makhubela, B. C. E.; Jardine, A.; Smith, G. S. Green Chem. 2012, $14,338$.

[76] Tharun, J.; Hwang, Y.; Roshan, R.; Ahn, S.; Kathalikkattil, A. C.; Park, D.-W. Catal. Sci. Technol. 2012, 2, 1674.

[77] Sun, J.; Wang, J.; Cheng, W.; Zhang, J.; Li, X.; Zhang, S.; She, Y. Green Chem. 2012, 14, 654.

[78] Tharun, J.; Kim, D.-W.; Roshan, R.; Hwang, Y.; Park, D.-W. Catal. Commun. 2013, 31, 62 .

[79] Gabriele, B.; Mancuso, R.; Salerno, G. Eur. J. Org. Chem. 2012, 6825.

[80] Liu, J.; Sun, W.; Zheng, S.; Xia, C. Helv. Chim. Acta 2007, 90, 1593.

[81] Xiang, Y.; Zhang, Q.; Si, J.; Du, J.; Guo, H.; Zhang, T. J. Mol. Catal. A: Chem. 2010, 322, 33.

[82] Chang, Y.-C.; Chen, D.-H. React. Funct. Polym. 2009, 69, 601.

[83] Chandrasekhar, V.; Athimoolam, A.; Srivatsan, S. G.; Sundaram, P. S.; Verma, S.; Steiner, A.; Zacchini, S.; Butcher, R. Inorg. Chem. 2002, 41, 5162.

[84] Srivatsan, S. G.; Verma, S. Chem.-Eur. J. 2001, 7, 828. 